\title{
HARMONIC MAJORIZATION OF SUBHARMONIC FUNCTIONS IN UNBOUNDED DOMAINS
}

\author{
S. J. GARDINER
}

\section{Introduction}

Let $\Omega$ be a domain in Euclidean space $\mathbf{R}^{n}$, where $n \geqq 2$. Its closure and boundary in $\mathbf{R}^{n}$ are respectively denoted by $\bar{\Omega}$ and $\partial \Omega$. We define a class of functions on $\bar{\Omega}$ by saying that $s \in \mathscr{S}(\bar{\Omega})$ if

(i) $s$ is defined on $\bar{\Omega}$,

(ii) $s$ is subharmonic in $\Omega$,

(iii) $\limsup _{\substack{X \rightarrow Y \\ X \in \Omega}} s(X)=s(Y)<+\infty \quad(Y \in \partial \Omega)$.

We note immediately that if $s \in \mathscr{S}(\bar{\Omega})$, then $s<+\infty$ on $\bar{\Omega}, s$ is upper semicontinuous (u.s.c.) on $\bar{\Omega}$ and so locally bounded above thereon.

First consider the case where $\Omega$ is bounded. (We will use the notation of Helms [12, Chapter 8] for concepts related to the Perron-Wiener-Brelot solution of the generalized Dirichlet problem.) If $s \in \mathscr{S}(\bar{\Omega})$ then, since $s$ is bounded above in $\bar{\Omega}$, it follows that $s \in \mathscr{L}_{s}$ and that $\mathscr{U}_{s}$ contains a finite constant function. Thus

$$
s \leqq \underline{H}_{s}^{\Omega} \leqq \bar{H}_{s}^{\Omega}<+\infty
$$

in $\Omega$. But, since the restriction of $s$ to $\partial \Omega$ is u.s.c., $\bar{H}_{s}^{\Omega}=\underline{H}_{s}^{\Omega}$ from [12, Theorem 8.13], and so $H_{s}^{\Omega}$ exists and is a harmonic majorant of $s$ in $\Omega$.

However, if $\Omega$ is unbounded and $s \in \mathscr{S}(\bar{\Omega})$, it need no longer be the case that $s$ has a harmonic majorant in $\Omega$. An obvious step is to take an expanding sequence $\left(\Omega_{m}\right)$ of bounded subdomains of $\Omega$ such that $\cup \Omega_{m}=\Omega$, and to consider the limit of the increasing sequence of harmonic functions $\left(H_{s}^{\Omega_{m}}\right)$. Unfortunately, except in the very simplest of cases, the expression for the harmonic measure of $\Omega_{m}$ will be too complicated to give a criterion for harmonic majorization in $\Omega$ that could be considered either elegant or useful. Instead, research in the half-space (Kuran [13] and Armitage [3]) and infinite strip (Brawn [8] and Armitage and Fugard [5]) has

This work was supported by a grant from the Department of Education for Northern Ireland. 
shown that special "means" over the boundaries of suitable subdomains of $\Omega$ can be used. The general outline of the methods in [5] may be employed in other domains but each case would require separate treatment. The purpose of this paper is to develop a fairly general theory, of which the above examples are actually special cases. The general results are not difficult to prove once a suitable expression for the mean has been identified (see $\mathscr{M}(s, x)$ below). The great benefit is that, having established these, applications to individual domains can be made very quickly for example, contrast $\S 8$ with [3] or $\S 9$ with [5].

\section{General results}

2.1. We denote points of $\mathbf{R}^{n}$ by $X, Y, Z, P$ or $Q$. When appropriate, $X$ will be written in terms of its co-ordinates

$$
X=\left(x_{1}, \ldots, x_{n}\right)=\left(X^{\prime}, x_{n}\right)
$$

where $X^{\prime} \in \mathbf{R}^{n-1}$. We recall that a bounded domain $\omega \in \mathbf{R}^{n}$ is called a Lipschitz domain if $\partial \omega$ can be covered by right circular cylinders whose bases have positive distance from $\partial \omega$, and corresponding to each cylinder $L$, there is a co-ordinate system $\left(\tilde{X}^{\prime}, \tilde{x}_{n}\right)$ with $\tilde{x}_{n}$-axis parallel to the axis of $L$, a function $\varphi: \mathbf{R}^{n-1} \rightarrow \mathbf{R}$ and a real number $c$ such that

for all $\tilde{X}^{\prime}, \tilde{Y}^{\prime} \in \mathbf{R}^{n-1}$,

$$
\left|\varphi\left(\tilde{X}^{\prime}\right)-\varphi\left(\tilde{Y}^{\prime}\right)\right| \leqq c\left|\tilde{X}^{\prime}-\tilde{Y}^{\prime}\right|
$$

and

$$
L \cap \omega=\left\{X \in L: \tilde{x}_{n}>\varphi\left(\tilde{X}^{\prime}\right)\right\},
$$

$$
L \cap \partial \omega=\left\{X \in L: \tilde{x}_{n}=\varphi\left(\tilde{X}^{\prime}\right)\right\} .
$$

From now on $\Omega$ will always denote an unbounded domain. Let $v$ be a fixed Radon measure with compact support $E \subset \bar{\Omega}$, and suppose that for each positive real number $x$ there is a corresponding open set $W_{x}$ whose intersection $\Omega_{x}$ with $\Omega$ is a bounded Lipschitz domain satisfying

(a) $E \subseteq \cap \bar{\Omega}_{x}$,

(b) $\bar{\Omega}=\cup \bar{\Omega}_{x}$,

(c) $x<y \Rightarrow \bar{\Omega}_{x} \subset W_{y} \cap \bar{\Omega}$.

The sets $\partial \Omega_{x} \cap \Omega$ and $\partial \Omega_{x} \cap \partial \Omega$ will be abbreviated to $\sigma_{x}$ and $\tau_{x}$, and $\mu_{x, X}$ will be used to denote harmonic measure on $\partial \Omega_{x}$ with respect to $X \in \Omega_{x}$.

If $s \in \mathscr{S}(\bar{\Omega})$, then, for each positive real number $x$, let

$$
\begin{gathered}
H_{s, x}(X)=H_{s}^{\Omega_{x}}(X)-\int_{\tau_{1}} s(X) d \mu_{1, X}(Y), \\
I_{s, x}(X)=\int_{\sigma_{x}} s(Y) d \mu_{x, X}(Y),
\end{gathered}
$$


and

$$
K_{s, x}(X)=\int_{\tau_{x} \backslash \tau_{1}} s(Y) d \mu_{x, X}(Y),
$$

which are clearly harmonic in $\Omega_{\min (x, 1)}$. Let $h_{*}$ denote a strictly positive harmonic function in $\Omega_{1}$ which vanishes on $\tau_{1}$ (clearly such functions exist, for example the Green function for $\Omega_{2}$ with its pole in $\Omega_{2} \backslash \Omega_{1}$; this is a consequence of the regularity of $\Omega_{2}$, which follows from the Zaremba cone criterion, [12, Theorem 8.27]).

Lemma 1. The quotients $H_{s, x} / h_{*}, I_{s, x} / h_{*}$ and $K_{s, x} / h_{*}$ can be continuously extended to $W_{y} \cap \bar{\Omega}$, where $y=\min (x, 1)$.

The extended functions of Lemma 1 will be denoted respectively by $\mathscr{H}_{s, x}, \mathscr{I}_{s, x}$ and $\mathscr{K}_{s, x}$ and are used to define

$$
\begin{aligned}
& \mathscr{M}(s, x)=\int_{E} \mathscr{H}_{s, x}(X) d v(X), \\
& \mathscr{N}(s, x)=\int_{E} \mathscr{I}_{s, x}(X) d v(X), \\
& \mathscr{P}(s, x)=\int_{E} \mathscr{K}_{s, x}(X) d v(X) .
\end{aligned}
$$

2.2. The following result is now almost immediate.

Theorem 1. (i) If $s \in \mathscr{S}(\bar{\Omega})$, then $\mathscr{M}(s, x)$ is an increasing, real-valued function of $x$.

(ii) If also $s \leqq 0$ on $\partial \Omega$, then the same is true of $\mathscr{N}(s, x)$.

(iii) If $h$ is harmonic in $\Omega$ and continuous on $\bar{\Omega}$, then $\mathscr{M}(h, x)$ is a constant function of $x$.

Theorem 2. If $s \in \mathscr{S}(\bar{\Omega})$ and $\mathscr{M}(s, x)$ is bounded above on $(0,+\infty)$, then $s$ has a harmonic majorant in $\Omega$.

It will be shown later that the converse of Theorem 2 is false. However, defining $\mathscr{T}(\bar{\Omega})$ to be the class of functions subharmonic in an open set containing $\bar{\Omega}$, we obtain the following.

Theorem 3. Let $s \in \mathscr{T}(\bar{\Omega})$. Then $s$ has a harmonic majorant in $\Omega$ if and only if $\mathscr{M}(s, x)$ is bounded above on $(0,+\infty)$.

Corollary. Let $s \in \mathscr{T}(\bar{\Omega})$ and $s \geqq 0$ in $\Omega$. Then $s$ has a harmonic majorant in $\Omega$ if and only if $\mathscr{N}(s, x)$ and $\mathscr{P}(s, x)$ each tend to a finite limit as $x \rightarrow+\infty$.

Finally we give two results which are very simple to prove.

Theorem 4. If $h$ is non-negative and harmonic in $\Omega$ and continuous in $\bar{\Omega}$, then

$$
\lim _{x \rightarrow+\infty} \mathscr{P}(h, x)<+\infty .
$$


Theorem 5. If $s \in \mathscr{S}(\bar{\Omega}), s \leqq 0$ on $\partial \Omega$ and

$$
\liminf _{x \rightarrow+\infty} \mathscr{N}\left(s^{+}, x\right)=0
$$

then $s \leqq 0$ in $\Omega$.

Theorem 5 is a generalization of a Phragmén-Lindelöf type of result (for example, see [1, Corollary to Theorem 1] and [14, Theorem 4]). One generalization of this type of theorem has already been given in [4] for arbitrary unbounded domains. There the mean employed is given by

$$
\lambda_{m}\left(s^{+}\right)=\liminf _{X \rightarrow P} I_{s, m}(X) / G_{\Omega_{1}}(Q, X),
$$

where $\left(\Omega_{m}\right)$ is an expanding sequence of bounded domains such that $\cup \Omega_{m}=\Omega$, and where we denote by $P$ either a point of $\Omega_{1}$ or a minimal Martin boundary point of $\Omega_{1}$, by $Q$ a fixed point in $\Omega_{1} \backslash\{P\}$, and by $G_{\Omega_{1}}$ the Green kernel of $\Omega_{1}$. It will later be clear that for certain domains $\Omega$ such as the whole space, half-space, and infinite cone, where the natural choice of $E$ is a singleton, $\lambda_{m}\left(s^{+}\right)$and $\mathscr{N}\left(s^{+}, m\right)$ can coincide. However, for other domains such as the infinite strip and infinite cylinder, these means differ significantly, and $\mathscr{N}\left(s^{+}, x\right)$ appears to be more natural, coinciding (in the case of the strip) with means that have previously been studied, [5].

We point out that if $E=\{P\} \subset \Omega$ and one of the subdomains, which we label as $\Omega_{1}$, satisfies $\bar{\Omega}_{1} \subset \Omega$, then the mean $\mathscr{M}(s, x)$ is merely a multiple of $H_{s}^{\Omega_{x}}(P)$.

The results of this section will be proved in $\S \S 3-6$.

\section{Proofs of Lemma 1 and Theorem 1}

3.1. We will make use of the following results.

Theorem A. Let $\Omega^{\prime}$ be a bounded Lipschitz domain and $f$ be a resolutive boundary function on $\partial \Omega^{\prime}$. If $f$ is continuous (in the extended sense) at a point $Y \in \partial \Omega^{\prime}$, then

$$
\lim _{X \rightarrow Y} H_{f}^{\Omega^{\prime}}(X)=f(Y) .
$$

Theorem B. If $h_{1}$ and $h_{2}$ are positive harmonic functions on a bounded Lipschitz domain $\Omega^{\prime}$ vanishing on a relatively open subset $A$ of $\partial \Omega^{\prime}$, then $h_{1} / h_{2}$ can be continuously extended to a strictly positive function defined on $\Omega^{\prime} \cup A$.

Theorem A is due to Armitage [2, Theorem 2]. Note that it asserts more than the regularity of $\Omega^{\prime}$. Theorem B is a slight modification of [6, Theorem 2].

3.2. We now prove Lemma 1. First we show that $H_{s, x}$ vanishes on $W_{y} \cap \partial \Omega$ If $x \geqq 1$, then define

$$
F(X)=\left\{\begin{array}{lll}
H_{s}^{\Omega_{x}}(X) & \text { if } & X \in \Omega_{x} \\
s(X) & \text { if } & X \in \partial \Omega_{x}
\end{array} .\right.
$$


From $[9$, p. $98(\varepsilon)]$ it follows that $H_{F}^{\Omega_{1}}=H_{s}^{\Omega_{x}}$ in $\Omega_{1}$, and so

$$
H_{s, x}(X)=H_{s}^{\Omega_{x}}(X)-\int_{\tau_{1}} s(Y) d \mu_{1, X}(Y)=\int_{\sigma_{1}} F(Y) d \mu_{1, X}(Y)
$$

Alternatively, if $x<1$, then similarly

$$
H_{s, x}(X)=\int_{\sigma_{x}}\left\{s(Y)-\int_{\tau_{1}} s(Z) d \mu_{1, Y}(Z)\right\} d \mu_{x, X}(Y) .
$$

Thus, in either case, $H_{s, x}$ is a harmonic function in $\Omega_{y}$ which, by Theorem A, vanishes on $W_{y} \cap \partial \Omega$.

If we now rewrite (2) as

$$
H_{s, x}(X)=\int_{\sigma_{1}} F^{+}(Y) d \mu_{1, X}(Y)-\int_{\sigma_{1}} F^{-}(Y) d \mu_{1, X}(Y),
$$

and treat (3) analogously, it follows by two applications of Theorem B that $H_{s, x} / h_{*}$ can be continuously extended to $W_{y} \cap \bar{\Omega}$.

In the case of the quotients $I_{s, x} / h_{*}$ and $K_{s, x} / h_{*}$, only the latter part of the above argument is required.

3.3. Theorem 1 is straightforward to prove. If $w<x$, then, with $F$ as in $\S 3.2$,

$$
H_{s}^{\Omega_{w}}(X) \leqq H_{F}^{\Omega_{w}}(X)=F(X) \quad\left(X \in \Omega_{w}\right)
$$

and so $\mathscr{M}(s, x)$ is an increasing function of $x$. If also $s \leqq 0$ on $\partial \Omega$, then

$$
\int_{\tau_{x}} s(Y) d \mu_{x, X}(Y) \leqq \int_{\tau_{w}} s(Y) d \mu_{w, X}(Y)
$$

since $\mu_{x, X} \geqq \mu_{w, X}$ on $\tau_{w}$, and so $I_{s, x} \geqq I_{s, w}$ in $\Omega_{w}$, proving (ii). Part (iii) is trivial.

\section{Proof of Theorem 2}

4.1. The following results will be required.

Theorem C. Let $\Omega^{\prime}$ be a bounded Lipschitz domain of which $P$ is a fixed point, $A$ be a relatively open subset of $\partial \Omega^{\prime}$, and $W^{\prime}$ be a subdomain of $\Omega^{\prime}$ satisfying $\partial \Omega^{\prime} \cap \partial W^{\prime} \subseteq A$. Then there is a constant $c$ such that, if $h_{1}$ and $h_{2}$ are two positive harmonic functions in $\Omega^{\prime}$ vanishing on $A$ and $h_{1}(P)=h_{2}(P)$, then $h_{1}(X) \leqq c h_{2}(X)$ for all $X \in W^{\prime}$.

Lemma 2. Let $s \in \mathscr{S}(\bar{\Omega})$ and define

Then $s_{x} \in \mathscr{S}(\bar{\Omega})$.

$$
s_{x}(X)=\left\{\begin{array}{lll}
H_{s}^{\Omega_{x}}(X) & \text { if } & X \in \Omega_{x} \\
s(X) & \text { if } & X \in \bar{\Omega} \backslash \Omega_{x} .
\end{array}\right.
$$


Theorem C can be found in [10, Theorem 4] or [17, Theorem 1]. The proof of Lemma 2 is very similar to that of [5, Lemma 2], but is given below for the sake of completeness.

To establish the subharmonicity of $s_{x}$ in $\Omega$, it suffices to show upper semicontinuity and the mean-value inequality at points of $\sigma_{x}$. Since $\Omega_{x}$ is regular and the restriction of $s$ to $\partial \Omega_{x}$ is u.s.c. and bounded above,

$$
\limsup _{X \rightarrow Y} H_{s}^{\Omega_{x}}(X) \leqq s(Y) \quad\left(Y \in \partial \Omega_{x}\right)
$$

[12, Lemma 8.20, Theorem 8.22]. Upper semicontinuity is now proved, and the mean-value inequality is an immediate consequence of the fact that $s \leqq H_{s}^{\Omega_{x}}$ in $\Omega_{x}$. In fact, combining the latter inequality with (4) yields

whence $s_{x} \in \mathscr{S}(\bar{\Omega})$.

$$
\limsup _{X \rightarrow Y} s_{x}(X)=s(Y)=s_{x}(Y) \quad(Y \in \partial \Omega)
$$

4.2. We will now prove Theorem 2. For each positive integer $k$ let $s_{k}$ be as in Lemma 2. From Theorem 1 (i) and the hypothesis of Theorem 2 it follows that

$$
\mathscr{M}\left(s_{k}, 1\right) \leqq \mathscr{M}\left(s_{k}, k\right)=\mathscr{M}(s, k) \leqq \sup _{k} \mathscr{M}(s, k)<+\infty .
$$

Since, for each $k,\left(s_{i}\right)_{i \geqq k}$ is an increasing sequence of harmonic functions in $\Omega_{k}$, either $\lim s_{i}$ is harmonic in $\Omega$ or is identically equal to $+\infty$. The theorem will follow if the latter case is shown not to hold.

Suppose $\lim s_{i} \equiv+\infty$. Then there exists $k_{0}$ such that $s_{k_{0}}>s_{2}$ in $\Omega_{2}$. Fixing $P \in \Omega_{1}$, Theorem $C$ shows that there is a positive constant $c$ such that, for $k \geqq k_{0}$ and $X \in \Omega_{1 / 2}$,

and so

$$
H_{s_{k}, 1}(X)-H_{s_{2}, 1}(X) \geqq c\left\{h_{*}(X) / h_{*}(P)\right\}\left\{H_{s_{k}, 1}(P)-H_{s_{2}, 1}(P)\right\}
$$

$$
\mathscr{M}\left(s_{k}, 1\right)-\mathscr{M}\left(s_{2}, 1\right) \geqq c^{\prime} \int_{\sigma_{1}}\left\{s_{k}(Y)-s_{2}(Y)\right\} d \mu_{1, P}(Y) \rightarrow+\infty \quad(k \rightarrow+\infty) .
$$

This contradicts (5) and so the result follows.

\section{Proof of Theorem 3 and Corollary}

5.1. The "if" part is contained in Theorem 2 since $\mathscr{T}(\bar{\Omega}) \subseteq \mathscr{S}(\bar{\Omega})$.

5.2. Conversely, $s$ is subharmonic in an open set $W$ containing $\bar{\Omega}$ and has a harmonic majorant in $\Omega$. It follows (compare [5, Lemma 3]) that the function $s_{0}$, equal in $\Omega$ to the least harmonic majorant of $s$ there, and equal in $W \backslash \Omega$ to $s$, is also subharmonic in $W$. From $[9$, Chapter IX, § 6],

$$
H_{s}^{\Omega_{x}}(X) \leqq H_{s_{0}}^{\Omega_{x}}(X)=s_{0}(X) \quad\left(X \in \Omega_{x}\right),
$$


whence

as required.

$$
\mathscr{M}(s, x) \leqq \mathscr{M}\left(s_{0}, x\right)=\mathscr{M}\left(s_{0}, 1\right) \quad(x \in(0,+\infty)),
$$

5.3. We come now to the proof of the Corollary. If $x \geqq 1$, define

$$
L_{s, x}(X)=\int_{\tau_{1}} s(Y)\left\{d \mu_{x, X}(Y)-d \mu_{1, X}(Y)\right\}
$$

and observe that $L_{s, x} / h_{*}$ may be continuously extended to $W_{1} \cap \bar{\Omega}$ (compare Lemma 1 ). Denoting the integral of this function with respect to $v$ by $\mathscr{Q}(s, x)$, it follows that

$$
\mathscr{M}(s, x)=\mathscr{N}(s, x)+\mathscr{P}(s, x)+\mathscr{Q}(s, x)
$$

Since $s \geqq 0$ and $\mu_{y, X} \geqq \mu_{x, X}$ on $\tau_{x}$ whenever $y>x$, it is easily seen that both $\mathscr{P}(s, x)$ and $\mathscr{Z}(s, x)$ increase with $x$. Also, $s$ has a positive upper bound, $c$ say, on $\bar{\Omega}_{1}$, whence

$$
L_{s, x}(X) \leqq c-c \mu_{1, X}\left(\tau_{1}\right)=c \mu_{1, X}\left(\sigma_{1}\right) \leqq c^{\prime} h_{*}(X) \quad\left(X \in \Omega_{1 / 2}\right)
$$

by Theorem $\mathrm{C}$. Hence $\mathscr{2}(s, x)$ is bounded above for all $x$ and so has a finite limit as $x \rightarrow+\infty$.

Now suppose that $\mathscr{N}(s, x)$ and $\mathscr{P}(s, x)$ each tend to a finite limit as $x \rightarrow+\infty$. Since this is also true of $\mathscr{Q}(s, x)$, it follows from (6) that $\mathscr{M}(s, x)$ is bounded above and so, by Theorem $3, s$ has a harmonic majorant in $\Omega$.

Conversely, suppose that $s$ has a harmonic majorant in $\Omega$. Then, using Theorems 1 and 3 and equation (6), it can be seen that $\mathscr{N}(s, x)+\mathscr{P}(s, x)$ tends to a finite limit as $x \rightarrow+\infty$. Since $\mathscr{P}(s, x) \geqq 0$ and $\mathscr{P}(s, x)$ is increasing, both $\mathscr{N}(s, x)$ and $\mathscr{P}(s, x)$ tend to a finite limit as $x \rightarrow+\infty$.

\section{Proofs of Theorems 4 and 5}

6.1. To prove Theorem 4, we note from Theorem 1 (iii) that $\mathscr{M}(h, x)$ is independent of $x$. Since $h \geqq 0$, it follows from (6) that $\mathscr{P}(h, x)$ is (increasing and) bounded above, whence the result.

6.2. To prove Theorem 5, first observe that $s^{+} \in \mathscr{S}(\bar{\Omega})$ and $s^{+}=0$ on $\partial \Omega$. Thus, from Theorem 1 (ii) and (1), $\mathscr{N}\left(s^{+}, x\right)=0$ for all $x$. In view of Theorem $\mathrm{B}$, it follows that $H_{s^{+}}^{\Omega_{x}} \equiv 0$ in $\Omega_{x}$ for all $x$. Since $s^{+} \leqq H_{s^{+}}^{\Omega_{x}}$ in $\Omega_{x}$, we have $s^{+} \equiv 0$ in $\Omega$, as required. 


\section{Applications to $\mathbf{R}^{n}$}

Let $\Omega=\mathbf{R}^{n}(n \geqq 2)$. The results in this case are elementary, but are given for the purpose of illustration. Let $E=\{O\}, v$ be the Dirac measure at the origin, $\Omega_{x}=B(x)$, the ball of radius $x$ centred at the origin, and take $h_{*} \equiv 1$ in $\mathbf{R}^{n}$. Clearly the hypotheses of $\S 2.1$ are satisfied. Since $\tau_{1}$ is empty, it is immediate that

$$
\mathscr{M}(s, x)=\mathscr{N}(s, x)=\left\{c_{n} x^{n-1}\right\}^{-1} \int_{\partial B(x)} s(Y) d \sigma(Y),
$$

where $c_{n}$ denotes the surface area of the unit sphere in $\mathbf{R}^{n}$, and $\sigma$ denotes surface area measure. From $\S 2$ we give immediately the following well-known results.

Theorem 6. Let $s$ be subharmonic in $\mathbf{R}^{n}(n \geqq 2)$.

(i) The mean $\mathscr{M}(s, x)$ is an increasing function of $x \in(0,+\infty)$;

(ii) $s$ has a harmonic majorant in $\mathbf{R}^{n}$ if and only if $\mathscr{M}(s, x)$ is bounded above on $(0,+\infty)$;

(iii) if $\lim \inf _{x \rightarrow+\infty} \mathscr{M}\left(s^{+}, x\right)=0$, then $s \leqq 0$ in $\mathbf{R}^{n}$.

\section{Applications to the half-space}

Let $\Omega=\mathbf{R}^{n-1} \times(0,+\infty)(n \geqq 2)$ and let $E$ and $v$ be as in $\S 7, \Omega_{x}=B(x) \cap \Omega$ and $h_{*}(X)=x_{n}$ in $\Omega$. Again the hypotheses of $\S 2.1$ are satisfied.

In order to find $\mathscr{M}(s, x)$ we recall (see (6)) that, if $x \geqq 1$,

$$
\mathscr{M}(s, x)=\mathscr{N}(s, x)+\mathscr{P}(s, x)+\mathscr{Q}(s, x) .
$$

As in [4, Example 2] it can be shown that

$$
\mathscr{N}(s, x)=\left(2 n / c_{n}\right) x^{-n-1} \int_{\sigma_{x}} y_{n} s(Y) d \sigma(Y)
$$

From the expression for the half-ball Poisson kernel given in [13, p. 615] it follows that

and

$$
\mathscr{P}(s, x)=\lim _{X \rightarrow O}\left(2 / c_{n}\right) \underset{\tau_{x} \backslash \tau_{1}}{\int} s(Y)\left\{|X-Y|^{-n}-x^{n}|Y|^{-n}\left|X-Y_{x}\right|^{-n}\right\} d \sigma(Y),
$$

$$
\mathscr{Q}(s, x)=\lim _{X \rightarrow 0}\left(2 / c_{n}\right) \int_{\tau_{1}} s(Y)\left\{|Y|^{-n}\left|X-Y_{1}\right|^{-n}-x^{n}|Y|^{-n}\left|X-Y_{x}\right|^{-n}\right\} d \sigma(Y),
$$

where $Y_{x}$ denotes the image of $Y$ under the inversion of centre $O$ and radius $x$. If $s \in \mathscr{S}(\bar{\Omega})$, then $s$ is locally integrable on $\partial \Omega$ (see [14, Theorem 1 (ii)]), and it is easy to check that the convergence of both integrands above is dominated by an 
integrable function. Hence

$$
\begin{gathered}
\mathscr{P}(s, x)+\mathscr{Q}(s, x)=\left(2 / c_{n}\right)\left\{\int_{\tau_{x} \backslash \tau_{1}} s(Y)\left\{|Y|^{-n}-x^{-n}\right\} d \sigma(Y)+\int_{\tau_{1}} s(Y)\left\{1-x^{-n}\right\} d \sigma(Y)\right\} \\
=\left(2 n / c_{n}\right) \int_{1}^{x} t^{-n-1} \int_{\tau_{t}} s(Y) d \sigma(Y) d t,
\end{gathered}
$$

using integration by parts (see $[14$, p. 314]). Thus

$$
\mathscr{U}(s, x)=\left(2 n / c_{n}\right)\left\{x^{-n-1} \int_{\sigma_{x}} y_{n} s(Y) d \sigma(Y)+\int_{1}^{x} t^{-n-1} \int_{\tau_{t}} s(Y) d \sigma(Y) d t\right\},
$$

and the same formula may similarly be established for $0<x<1$.

Further, if $s \geqq 0$ in $\bar{\Omega}$ then the statement $\lim _{x \rightarrow+\infty} \mathscr{P}(s, x)<+\infty$ holds if and only if

To see this,

$$
\int_{\partial \Omega}\left(1+|Y|^{2}\right)^{-n / 2} s(Y) d \sigma(Y)<+\infty
$$

$$
\begin{gathered}
\frac{1}{2} c_{n} \lim _{x \rightarrow+\infty} \mathscr{P}(s, x)=\lim _{x \rightarrow+\infty} \int_{\tau_{x} \backslash \tau_{1}}\left(|Y|^{-n}-x^{-n}\right) s(Y) d \sigma(Y) \\
\geqq \lim _{x \rightarrow+\infty}\left(1-2^{-n}\right) \int_{\tau_{x / 2} \backslash \tau_{1}}|Y|^{-n} s(Y) d \sigma(Y) \geqq\left(1-2^{-n}\right) \int_{\partial \Omega \backslash \tau_{1}}\left(1+|Y|^{2}\right)^{-n / 2} s(Y) d \sigma(Y),
\end{gathered}
$$

proving the "only if" case. The converse is even more elementary.

In this context, Theorems 1 (i), (iii), 4 and 5 are given in [14], the Corollary to Theorem 3 is part of [13, Theorem 3], and Theorems 2, 3 are due to Armitage [3], who also provides a counterexample to the converse of Theorem $2,[3, \S 6]$.

\section{Applications to the infinite cylinder}

Recently results similar to those of $\S 2.2$ have been obtained in the infinite strip, [5], and the infinite cone, [11]. These can be shown to be special cases of our general theorems. However, for the sake of originality, we will deduce previously unpublished results for the infinite cylinder. Modifications of our methods can be employed in the above-mentioned cases.

Let $\Omega=\left\{X=\left(x_{1}, \ldots, x_{n}\right)=\left(X^{\prime}, x_{n}\right):\left|X^{\prime}\right|<1\right\}, \quad(n \geqq 2)$. We will employ the Bessel function $J_{(n-3) / 2}$ defined in Watson [16, pp. 40-42]. The least positive zero of this function will be denoted by $a_{n}$ and we write

and

$$
\psi(t)=t^{(3-n) / 2} J_{(n-3) / 2}\left(a_{n} t\right) \quad(t>0)
$$

$$
b_{n}=a_{n} J_{(n-1) / 2}\left(a_{n}\right)>0,
$$

(see [16, p. 45 (4) and p. $479 \S 15.22]$ ). We will require the following 
Lemma 3. The functions $\psi\left(\left|X^{\prime}\right|\right) \exp \left( \pm a_{n} x_{n}\right)$ are positive and harmonic in $\Omega$, and vanish on $\partial \Omega$.

The proof of Lemma 3 is analogous to that of [7, Lemma 1].

Now let

and

$$
\begin{gathered}
E=\left\{X \in \mathbf{R}^{n}:\left|X^{\prime}\right| \leqq 1, \quad x_{n}=0\right\}, \\
d v(X)=2 a_{n}\left\{\psi\left(\left|X^{\prime}\right|\right)\right\}^{2} d X^{\prime} d \delta_{0}\left(x_{n}\right) \quad(X \in E)
\end{gathered}
$$

$$
h_{*}(X)=\psi\left(\left|X^{\prime}\right|\right) \cosh \left(a_{n} x_{n}\right),
$$

where $\delta_{0}$ denotes the Dirac measure at the origin of $\mathbf{R}$. It follows from Lemma 3 that $h_{*}$ satisfies the hypotheses of $\S 2.1$. Also, let $\Omega_{x}=\left\{X \in \Omega:\left|x_{n}\right|<x\right\}$. If $s \in \mathscr{S}(\bar{\Omega})$, then

$$
\begin{gathered}
\mathscr{N}(s, x)=2 a_{n} \int_{\left\{\left|Y^{\prime}\right|<1\right\}}\left\{\int_{\sigma_{x}} s(X) d \mu_{x,\left(Y^{\prime}, 0\right)}(X)\right\} \psi\left(\left|Y^{\prime}\right|\right) d Y^{\prime} \\
=2 a_{n} \gamma_{n} \int_{\sigma_{x}} s(X)\left\{\int_{\left\{\left|Y^{\prime}\right|<1\right\}} \psi\left(\left|Y^{\prime}\right|\right) \frac{\partial}{\partial n_{X}} G_{\Omega_{x}}\left(X,\left(Y^{\prime}, 0\right)\right) d Y^{\prime}\right\} d \sigma(X),
\end{gathered}
$$

where $\gamma_{2}=(2 \pi)^{-1}, \gamma_{n}=\left\{(n-2) c_{n}\right\}^{-1}(n \geqq 3)$ (see [10, Theorem 3] or [15, Theorem C]), and $n_{X}$ denotes the inward unit normal at $X$ with respect to $\Omega_{x}$. The interchange in order of integration is justified because $s$ is integrable with respect to harmonic measure on the boundary of $\Omega_{x}$. Now, if $x_{n}=x$,

$$
\left(\partial / \partial n_{x}\right) G_{\Omega_{x}}\left(X,\left(Y^{\prime}, 0\right)\right)=\lim _{t \rightarrow x-} G_{\Omega_{x}}\left(\left(X^{\prime}, t\right),\left(Y^{\prime}, 0\right)\right) /(x-t)
$$

this convergence being dominated by a constant for all $\left|Y^{\prime}\right|<1$ (see Theorem $\mathrm{C}$ ). A similar argument (with a change of sign) holds for $x_{n}=-x$, and so

$$
\mathscr{N}(s, x)=\int_{\sigma_{x}} s(X)\left(\partial / \partial n_{x}\right) G_{\Omega_{x}} \mu(X) d \sigma(X)
$$

where

$$
d \mu(Y)=2 a_{n} \gamma_{n} \psi\left(\left|Y^{\prime}\right|\right) d Y^{\prime} d \delta_{0}\left(y_{n}\right) \quad(Y \in E) .
$$

Now consider the function

$$
u_{x}(X)=\sinh a_{n}\left(x-\left|x_{n}\right|\right) \operatorname{sech}\left(a_{n} x\right) \psi\left(\left|X^{\prime}\right|\right)
$$

which, as is easy to check from Lemma 3 , is positive and superharmonic in $\Omega_{x}$, harmonic in $\Omega_{x} \backslash\left\{x_{n}=0\right\}$ and continuously vanishes on $\partial \Omega_{x}$. It follows that $u_{x}$ is a potential in $\Omega_{x}$, and its corresponding measure is given by $\mu^{\prime}=-\gamma_{n} \Delta u_{x}$, where $\Delta u_{x}$ is the distributional Laplacian of $u_{x}$.

Now let $\Psi$ be a $C^{\infty}$ function with compact support in $\Omega_{x}$. From Green's 
theorem it follows that

$$
\begin{gathered}
\left(\Delta u_{x}\right)(\Psi)=\int_{\Omega_{x}} u_{x}(X) \Delta \Psi(X) d X \\
=\lim _{\varepsilon \rightarrow 0+}\left\{\int_{\left\{\left|X^{\prime}\right|<1, x_{n}=\varepsilon\right\}}\left\{\Psi(X) \frac{\partial u_{x}}{\partial x_{n}}(X)-u_{x}(X) \frac{\partial \Psi}{\partial x_{n}}(X)\right\} d \sigma(X)\right. \\
\left.+\int_{\left\{\left|X^{\prime}\right|<1, x_{n}=-\varepsilon\right\}}\left\{u_{x}(X) \frac{\partial \Psi}{\partial x_{n}}(X)-\Psi(X) \frac{\partial u_{x}}{\partial x_{n}}(X)\right\} d \sigma(X)\right\} \\
=-2 a_{n} \int_{\left\{\left|X^{\prime}\right|<1\right\}} \Psi\left(X^{\prime}, 0\right) \psi\left(\left|X^{\prime}\right|\right) d X^{\prime},
\end{gathered}
$$

whence $\mu=\mu^{\prime}$. Thus, from (7),

$$
\mathscr{N}(s, x)=a_{n} \operatorname{sech}\left(a_{n} x\right) \int_{\sigma_{x}} \psi\left(\left|X^{\prime}\right|\right) s(X) d \sigma(X) .
$$

The determination of the remaining part of $\mathscr{M}(s, x)$ is similar, and involves calculating the normal derivative of $u_{x}$ at points of $\tau_{x} \backslash\left\{\left|x_{n}\right|=0\right.$ or $\left.x\right\}$ (noting that the interchange of differentiation and integration to obtain (7) is valid except on the set $\tau_{x} \cap\left\{\left|x_{n}\right|=0\right.$ or $\left.x\right\}$ which has surface area measure zero), performing the corresponding calculation for $u_{1}$, and integrating by parts (as in $\S 8$ ) to obtain

$$
\mathscr{U}(s, x)=\mathscr{N}(s, x)+a_{n} b_{n} \int_{1}^{x} \operatorname{sech}^{2}\left(a_{n} t\right) \int_{\tau_{t}} s(X) \cosh \left(a_{n} x_{n}\right) d \sigma(X) d t .
$$

The results of $\S 2.2$ may now be applied to subharmonic functions in the infinite cylinder. In the case of Theorem 4 , it is a routine matter to check that the conclusion is equivalent to the simple condition

$$
\int_{\partial \Omega} \exp \left(-a_{n}\left|x_{n}\right|\right) h(X) d \sigma(X)<+\infty .
$$

The details are left to the reader.

\section{References}

[1] AhLFors, L. V.: Remarks on Carleman's formula for functions in a halfplane. - SIAM J. Numer. Anal. 3, 1966, 183-187.

[2] Armitage, D. H.: A strong type of regularity for the PWB solution of the Dirichlet problem. Proc. Amer. Math. Soc. 61, 1976, 285-289.

[3] Armitage, D. H.: Half-spherical means and harmonic majorization in half-spaces. - J. London Math. Soc. (2) 19, 1979, 457-464.

[4] Armitage, D. H.: A Phragmén-Lindelöf theorem for subharmonic functions. - Bull. London Math. Soc. 13, 1981, 421-428.

[5] Armitage, D. H., and T. B. Fugard: Subharmonic functions in strips. - J. Math. Anal. Appl. $89,1982,1-27$. 
[6] Armitage, D. H., and S. J. Gardiner: Some Phragmén-Lindelöf and harmonic majorization theorems for subharmonic functions. - J. Math. Anal. Appl. (to appear).

[7] Brawn, F. T.: Mean value and Phragmén-Lindelöf theorems for subharmonic functions in strips. - J. London Math. Soc. (2) 3, 1971, 689-698.

[8] BRAwn, F. T.: Positive harmonic majorization of subharmonic functions in strips. - Proc. London Math. Soc. (3) 27, 1973, 261-289.

[9] Brelot, M., Éléments de la théorie classique du potentiel. - Centre de documentation universitaire, Paris, 1965.

[10] Dahlberg, B. E. J.: Estimates of harmonic measure. - Arch. Rational Mech. Anal. 65, 1977, $275-288$.

[11] FugARD, T. B.: Harmonic and subharmonic functions in cones and half-spaces. - Doctoral thesis, The Queen's University of Belfast, 1981.

[12] HeLms, L. L.: Introduction to potential theory. - Wiley-Interscience, a division of John Wiley \& Sons, New York-London-Sydney-Toronto, 1969.

[13] Kuran, Ü.: Harmonic majorizations in half-balls and half-spaces. - Proc. London Math. Soc. (3) $21,1970,614-636$.

[14] Kuran, Ü.: On half-spherical means of subharmonic functions in half-spaces. - J. London Math. Soc. (2) 2, 1970, 305-317.

[15] KuRAn, Ü.: Dirichlet resolutivity and surface-area integrability. - Proc. London Math. Soc. (3) $26,1973,339-358$.

[16] Watson, G. N.: A treatise on the theory of Bessel functions. - Cambridge University Press, Cambridge, 1944.

[17] Wu, J.-M. G.: Comparisons of kernel functions, boundary Harnack principle and relative Fatou theorems on Lipschitz domains. - Ann. Inst. Fourier (Grenoble) 28: 4, (1978), $147-167$.

The Queen's University of Belfast Department of Pure Mathematics Belfast BT7 1NN

Northern Ireland

Received 25 February 1982
Present address

Department of Agriculture

Biometrics Division

Newforge Lane

Belfast BT9 5PX

Northern Ireland 